\title{
Automatic Detection and Segmentation of Ground Glass Opacity Nodules
}

\author{
Jinghao Zhou ${ }^{1}$, Sukmoon Chang ${ }^{1,2}$, Dimitris N. Metaxas ${ }^{1}$, Binsheng Zhao ${ }^{3}$, \\ Lawrence H. Schwartz ${ }^{3}$, and Michelle S. Ginsberg ${ }^{3}$ \\ ${ }^{1}$ CBIM, Rutgers, The State University of New Jersey, NJ, USA \\ jhzhou@eden.rutgers.edu, dnm@cs.rutgers.edu \\ ${ }^{2}$ Computer Science, Capital College, Penn State University, Middletown, USA \\ sukmoon@psu.edu \\ ${ }^{3}$ Department of Radiology, Memorial Sloan-Kettering Cancer Center, NY, USA \\ zhaob@mskcc.org, schwartl@mskcc.org, ginsberm@mskcc.org
}

\begin{abstract}
Ground Glass Opacity (GGO) is defined as hazy increased attenuation within a lung that is not associated with obscured underlying vessels. Since pure (nonsolid) or mixed (partially solid) GGO at the thinsection CT are more likely to be malignant than those with solid opacity, early detection and treatment of GGO can improve a prognosis of lung cancer. However, due to indistinct boundaries and inter- or intra-observer variation, consistent manual detection and segmentation of GGO have proved to be problematic. In this paper, we propose a novel method for automatic detection and segmentation of GGO from chest CT images. For GGO detection, we develop a classifier by boosting $k$-NN whose distance measure is the Euclidean distance between the nonparametric density estimates of two examples. The detected GGO region is then automatically segmented by analyzing the texture likelihood map of the region. We applied our method to clinical chest $\mathrm{CT}$ volumes containing 10 GGO nodules. The proposed method detected all of the 10 nodules with only one false positive nodule. We also present the statistical validation of the proposed classifier for GGO detection as well as very promising results for automatic GGO segmentation. The proposed method provides a new powerful tool for automatic detection as well as accurate and reproducible segmentation of GGO.
\end{abstract}

\section{Introduction}

Ground Glass Opacity (GGO) is defined as hazy increased attenuation within a lung that is not associated with obscured underlying vessels, but with preservation of bronchial and vascular margins [1. It can reflect minimal thickening of the septal or alveolar interstitium, thickening of alveolar walls, or the presence of cells or fluid filling the alveolar spaces. It can represent active disease such as pulmonary edema, pneumonia, or diffuse alveolar damage. The results of the Early Lung Cancer Action Project, or ELCAP, suggested that nodules with pure (nonsolid) or mixed (partially solid) GGO at the thin-section CT are more likely to be malignant than are those with solid opacity [2]. A focal area of 
pure GGO on the thin-section CT seems to be an early sign of bronchioloalveolar carcinoma (BAC) 3. Pure GGO is useful for differentiating small localized BAC from small adenocarcinomas not having a replacement growth pattern 4. Detection and treatment of pure GGO can improve a prognosis of lung cancer [5].

The appearances of GGO on CT images such as its shape, pattern, and boundary are very different from solid nodules. Thus, algorithms developed for segmentation of solid nodules are very likely to produce inaccurate results when applied to GGO. In [6], a hybrid neural network of three single nets and an expert rule are applied to detect GGO. This method underestimates GGO area due to its improper cut-off of the edges of GGO. Hence, this method may be used only for large GGO and may not be able to obtain accurate segmentation for small GGO. [7] detected GGO using automatic clustering techniques and focused only on GGO detection. The GGO segmentation was not discussed in their work. 8 applies GGO segmentation method based on Markov random field and vessel removal method based on shape analysis. However, they only focused on GGO segmentation. The GGO detection was not discussed in their work.

In this paper, we propose a novel method for automatic detection and segmentation of GGO from chest CT images. For GGO detection, we develop a classifier by boosting $k$-NN whose distance measure is the Euclidean distance between the nonparametric density estimates of two examples. The detected GGO region is then automatically segmented by analyzing the texture likelihood map of the region. We also present the statistical validation of the proposed classifier for GGO detection as well as the very promising results of automatic GGO segmentation. The proposed method provides a new powerful tool for automatic detection as well as accurate and reproducible segmentation of GGO.

\section{Method}

\subsection{GGO Detection}

We first develop a novel method for automatic GGO detection from chest CT images. The method is based on texture analysis using a machine learning framework, i.e., boosting the $k$-NN classifier. Our primary focus in this section is the accurate classification of GGO from other objects in chest CT images.

Vessel and noise suppression with Cylinder filters. The accuracy of GGO detection may be hindered by various structures within a lung. To avoid this difficulty, we first develop a cylinder filter to suppress intensity values of vessels and other elongated structures as well as noise inside a lung, while maintaining GGO intensity values intact 9. The cylinder filter $F_{\text {cyl }}$ is defined as:

$$
F_{\mathrm{cyl}}(x)=\min _{\theta}\left(\min _{y \in \Omega_{\theta}^{x}} I(y)\right)
$$

where, $\Omega_{\theta}^{x}$ is the domain of the cylinder filter centered at $x$ with orientation $\theta$. $F_{\text {cyl }}$ is a hybrid neighborhood proximity filter that produces strong responses to 


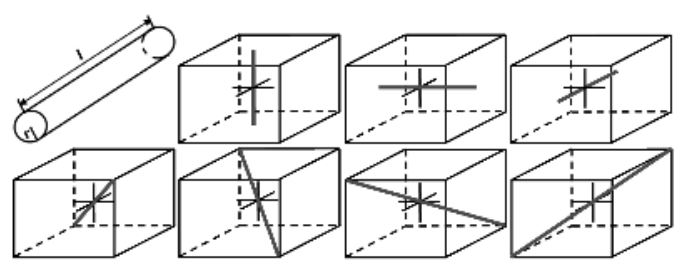

Fig. 1. Cylinder filter and its 7 orientations

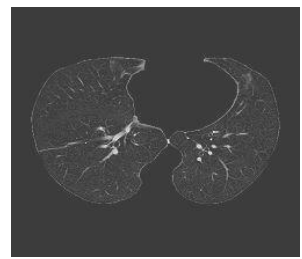

(a)

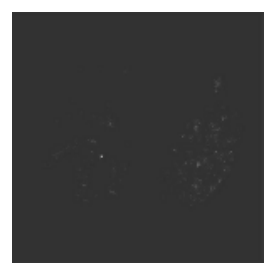

(b)

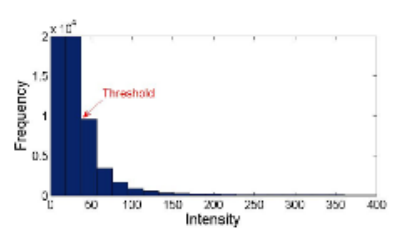

Fig. 2. Histogram of a cylinder filtered image

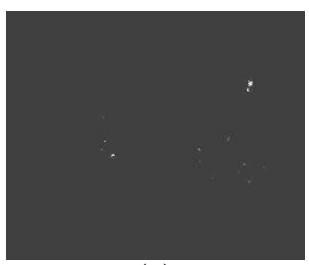

(c)

Fig. 3. Effect of $F_{\text {cyl }}$. (a) Original image (b) Image filtered with $F_{\text {cyl }}$ (c) After thresholding.

blob-like objects (i.e. GGO). In this paper, we have selected the parameters of $F_{\text {cyl }}$ empirically and used a cylinder with radius of 1,2 and 3 voxels and length of 7 voxels at 7 different orientations, as shown in Fig. 1. The effect of $F_{\text {cyl }}$ is shown in Fig. 3. In the figure, (b) shows the filter-response image of $F_{\text {cyl }}$ applied to (a). We can see from (b) that vessels and noise are effectively suppressed while GGO remains intact.

To isolate candidate GGO areas, i.e., areas of high response values, we apply a simple thresholding to the filter-response image (Fig. 3(c)). The threshold value is automatically determined by analyzing the histogram of the filter-response image, as illustrated in Fig. 2. In the next section, we apply a machine learning framework to classify the candidate GGO areas.

Classification of GGO by boosting $\boldsymbol{k}$-NN. $k$-NN assumes that an instance is represented as a point in $n$-dimensional space, $\mathcal{R}^{n}$. The nearest neighbors of an instance are defined in terms of the standard Euclidean distance. An instance, $x$, can be denoted by a feature vector, $\left\langle a_{1}(x), a_{2}(x), \ldots, a_{n}(x)\right\rangle$, where $a_{r}(x)$ denotes the value of the $r^{\text {th }}$ attribute of $x$. Then, the distance between two instances $x_{i}$ and $x_{j}$ is defined as:

$$
d\left(x_{i}, x_{j}\right)=\sqrt{\sum_{r=1}^{n}\left(a_{r}\left(x_{i}\right)-a_{r}\left(x_{j}\right)\right)^{2}}
$$

Fig. 4 shows the typical intensity pdf (probability density function) of positive and negative examples. We can see from the figure that positive and negative 
examples are well separable using the pdf. For this reason, we use as an instance the intensity pdf of the region surrounding a candidate GGO.

The learning for the classification of the possible GGO areas isolated from the previous section has a discrete target function of the form $f: \mathcal{R}^{n} \mapsto \mathcal{V}$, where $\mathcal{V}=\{\oplus, \ominus\}$, with the label $\oplus$ for GGO and $\ominus$ for non GGO areas. The $k$-NN algorithm returns $\hat{f}\left(x_{q}\right)$ as its estimate of $f\left(x_{q}\right)$, which is the most common value of $f$ among the $k$ training examples nearest to $x_{q}$ :

$$
\hat{f}\left(x_{q}\right)=\underset{v \in \mathcal{V}}{\arg \max } \sum_{i=1}^{k} \delta\left(v, f\left(x_{i}\right)\right)
$$

where, $x_{q}$ is a query instance to be classified, $x_{1}, \ldots, x_{k}$ denote the $k$ instances from training samples that are nearest to $x_{q}$, and $\delta(a, b)=1$ if $a=b$ and $\delta(a, b)=$ 0 otherwise. To obtain an accurate classification, $k$-NN needs a large training set, which results in slow classification due to the large number of distance calculations. We overcome this difficulty by boosting the $k$-NN.

Boosting is a method for improving the performance of any weak learning algorithm which, in theory, only needs to perform slightly better than random guessing. A boosting algorithm called AdaBoost improves the performance of a given weak learning algorithm by repeatedly running the algorithm on the training data with various distributions and then combining the classifiers generated by the weak learning algorithm into a single final classifier [10. As in [10], our purpose of using AdaBoost is to improve the speed of $k$-NN by reducing the number of prototype samples and thus reducing the required number of distance calculation without affecting the error rate. The details of boosting $k$-NN is given in 10

\subsection{GGO Segmentation in ROI}

Because of the hazy appearance of GGO and the large overlap of intensity values between GGO and surrounding vessels, simple thresholding or edge based segmentation method do not produce acceptable results for GGO segmentation. The proposed method applies a nonparametric density estimation and likelihood map method based on the texture of GGO for segmentation [1], followed by eigenanalysis of the Hessian matrix to remove vessels overlapped with GGO [12.

Nonparametric density estimation. Let $\mathcal{R}_{M}$ be the volumetric region of a candidate GGO bounded by a cube model $\Phi_{M}$. We estimate the probability density function (pdf) of intensity values of the model interior. We use a nonparametric kernel based method to approximate the pdf. Let $i, i=0, \ldots, 255$, denote the random variable for intensity values. The intensity pdf of the modelinterior region is defined by:

$$
P\left(i \mid \Phi_{M}\right)=\frac{1}{V\left(\mathcal{R}_{M}\right)} \iiint_{\mathcal{R}_{M}} \frac{1}{\sqrt{2 \pi \sigma^{2}}} \exp \left(-\frac{(i-I(y))^{2}}{2 \sigma^{2}}\right) d y
$$




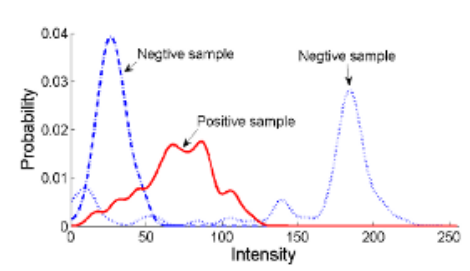

Fig. 4. Typical probability density functions of positive (solid), negative (dash-dot, typical background), and negative (dotted, typical vessels) examples

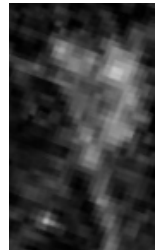

(a)

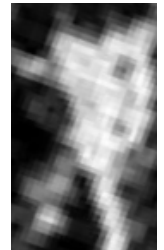

(b)

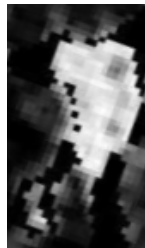

(c)
Fig. 5. (a) Original image of GGO, (b) likelihood map in ROI computed based on texon statistics method, and (c) Likelihood map in ROI after vessel removal based on eigenanalysis of the Hessian matrix

where, $V\left(\mathcal{R}_{M}\right)$ denotes the volume of $\mathcal{R}_{M}, y$ are the pixels in the domain $\mathcal{R}_{M}$, and $\sigma$ is the standard deviation of a Gaussian kernel.

We use an information theoretic distance measure called Kullback-Leibler Divergence (KLD) to measure the dissimilarity between 2 pdf's described in the previous section [13. The symmetrized variation of KLD is called Chernoff Information. The Chernoff Information between $p_{1}$ and $p_{2}$ is defined by:

$$
C\left(p_{1} \| p_{2}\right)=\max _{0 \leq t \leq 1}(-\log \mu(t))
$$

where, $\mu(t)=\int\left[p_{1}(i)\right]^{1-t}\left[p_{2}(i)\right]^{t} d i$. A special case of Chernoff distance is the Bhattacharya distance, in which $t$ is chosen to be $\frac{1}{2}$, i.e., the Bhattacharya distance between $p_{1}$ and $p_{2}$ is:

$$
B\left(p_{1} \| p_{2}\right)=-\log \mu\left(\frac{1}{2}\right)
$$

Also, we denote:

$$
\rho\left(p_{1} \| p_{2}\right)=\mu\left(\frac{1}{2}\right)=\int\left[p_{1}(i)\right]^{\frac{1}{2}}\left[p_{2}(i)\right]^{\frac{1}{2}} d i
$$

Eq. (11) and (2) shows that, when the value of $\rho$ goes from zero to one, the corresponding value of $B$ goes from infinity to zero. The Chernoff Information is an important information-theoretic distance measure, and it has been shown this measure is the exponential rate of optimal classifier performance probabilities 14 .

Texture likelihood map. We now evaluate the likelihood of the neighborhood sphere texon of every pixel in ROI. We define this likelihood using $\rho$, since it increases as the Bhattacharya distance between two distributions decreases. That is, the likelihood of any pixel $x$ belonging to the object of interest is defined by:

$$
L\left(T(x, \hat{s}) \mid \Phi_{M}\right) \propto \rho\left(p_{T(x, \hat{s})} \| p_{m}\right)
$$


where, $T(x, \hat{s})$ represents the neighborhood sphere texon centered at $x$ with scale $\hat{s}, p_{T(x, \hat{s})}$ is the intensity pdf of the texon, and $p_{m}$ is the learned pdf of $\Phi_{M}$. Fig. 5(b) shows the texture likelihood map of the image in (a). The radius of texons in our paper is less than 3 pixels and the model interior texture is mostly homogeneous with some level noise. Thus, it is not necessary to consider the spatial correlation between pixels.

Vessel removal. Finally, we remove the vessels around GGO in the texture likelihood map. The eigenanalysis of the Hessian matrix is a widely used method for vessel detection [12. Given an image $I(x)$, the local intensity variations in the neighborhood of a point $x_{0}$ can be expressed with its Taylor expansion:

$$
I\left(x_{0}+h\right) \approx I\left(x_{0}\right)+h^{T} \nabla I\left(x_{0}\right)+h^{T} H\left(x_{0}\right) h
$$

where, $\nabla I\left(x_{0}\right)$ and $H\left(x_{0}\right)$ denote the gradient and the Hessian matrix at $x_{0}$, respectively. $H\left(x_{0}\right)$, whose components are the second order derivatives, describes the curvature of $I(x)$ at $x_{0}$. Let $\lambda_{1}, \lambda_{2}, \lambda_{3}$ and $\boldsymbol{e}_{\mathbf{1}}, \boldsymbol{e}_{\mathbf{2}}, \boldsymbol{e}_{\mathbf{3}}$ be the eigenvalues and eigenvectors of $H$ such that $\lambda_{1} \leq \lambda_{2} \leq \lambda_{3}$ and $\left|\boldsymbol{e}_{\boldsymbol{i}}\right|=1$. The signs and ratios of the eigenvalues provide the indications of various shapes of interest, as summarized in [15]. Fig. [5)(c) shows the texture likelihood map after the removal of vessels in (b).

\section{Results}

To test the GGO detection method, we collected 319 example images, containing 200 training examples and 119 test examples. The example images were of size $9 \times$ 9 pixels extracted from the CT volumes. The distance that we used for $k$-NN to identify the nearest neighbor and to classify an instance is the standard Euclidean distance between the nonparametric density estimates (represented as vectors in $\mathcal{R}^{256}$ ) of two examples as described in Sec. 2.2. We performed bootstrapping to estimate the generalization error of our GGO detection method 16. We trained and tested the method on bootstrap samples. After 20 steps of boosting, the test error rate converges to $5.04 \%$.

We applied the trained GGO classifier to $10 \mathrm{CT}$ volumes containing 10 nodules. The CT volumes were acquired by multi-slice HRCT scanners with $1 \mathrm{~mm}$ slice collimation. The number of slices in each CT scan ranged from 23 to 29 (interpolated to 92 to 116 ), each of which consists of a $512 \times 512$ matrix, with in-plane resolution ranging from $0.57 \mathrm{~mm}$ to $0.71 \mathrm{~mm}$. The classifier detected the total of 11 nodules, containing all of the 10 nodules and one false positive nodule.

The detected nodules were then segmented as described in Sec. 2.2. Fig. 6 shows the GGO segmentation results overlaid on the original CT images and 3D reconstruction for three GGOs as examples. From the figure, we can see that the surrounding vessels are accurately removed from the GGO segmentation. These results demonstrate the potential of our segmentation method to deal with haze patterns of GGO. 

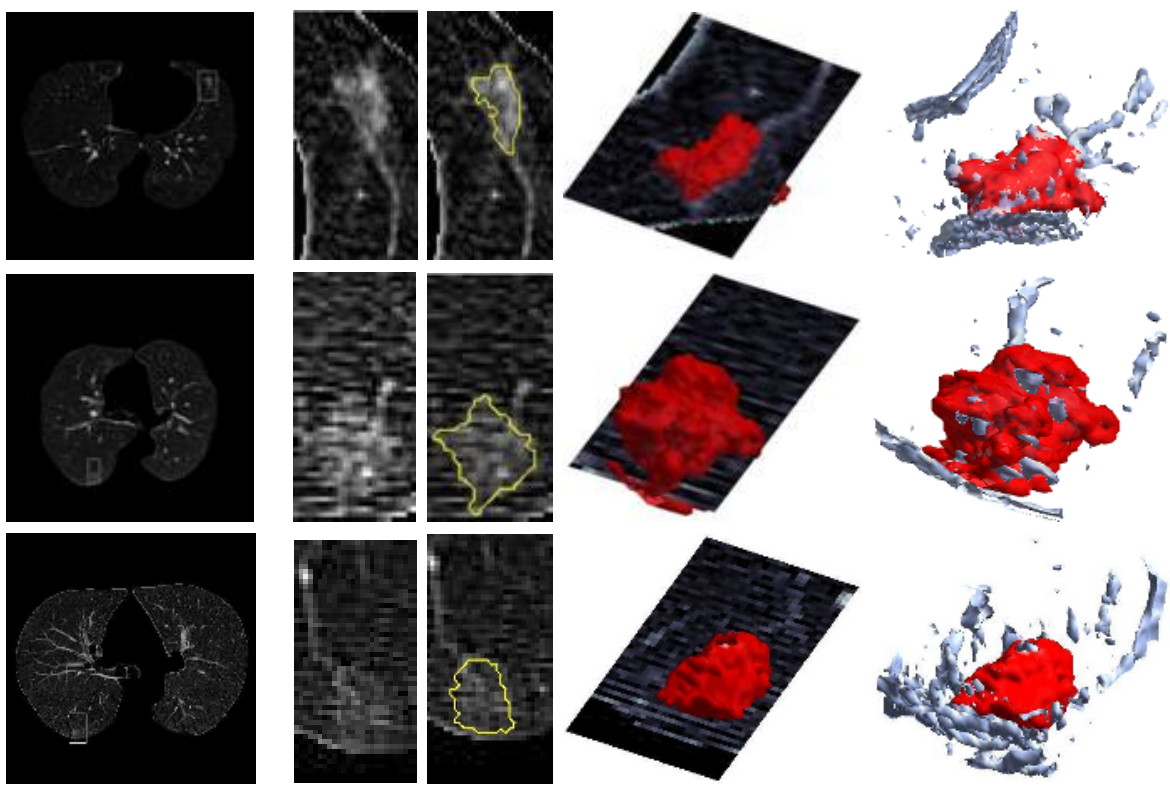

(d)

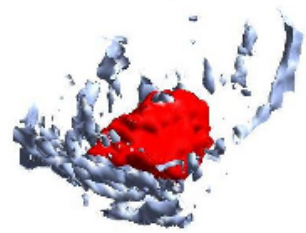

(e)

Fig. 6. GGO segmentation. (a) original CT images, (b) enlarged CT images, (c) segmentation overlay, (d)and (e) 3D reconstruction of segmented GGO

\section{Conclusion}

We proposed a novel method for the automatic detection and segmentation of GGO from chest CT images. The proposed method consists of two steps, i.e., GGO detection, followed by GGO segmentation. For GGO detection, vessels and noise were first suppressed using cylinder filters. Then, candidate GGO regions were extracted by thresholding. We automatically selected the threshold by the histogram analysis of the filter-response images. Finally, the candidate GGO regions were classified by boosting the $k$-NN, whose distance measure was the Euclidean distance between the intensity pdf's of two examples. The validation of the proposed method using bootstrapping shows the mean error rate of $5.04 \%$. Our method applied to clinical chest CT volumes containing 10 GGO nodules also shows the promissing result, detecting all of the 10 nodules with one false positive nodule.

Each GGO region identified was automatically segmented by analyzing the texture likelihood map of the region. We presented various results of GGO detection and segmentation from clinical chest CT images. The manual segmentation of GGO has proved to be problematic due to large inter-observer variations as well as large intra-observer variations. The proposed method introduces a novel automatic tool for accurate detection as well as reproducible segmentation of GGO. 


\section{References}

1. Austin, J., Muller, N., Friedman, P.e.a.: Glossary of terms for CT of the lung: recommendations of the nomenclature committee of the fleischner society. Radiology 200 (1996) 327-331

2. Henschke, C., Yankelevitz, D., Mirtcheva, R.: CT screening for lung cancer: frequency and significance of part-solid and nonsolid nodules. Am. J. Roentgenol. 178 (2002) 1053-1057

3. Jang, H., Lee, K., Kwon, O., Rhee, C., Shim, Y., Han, J.: Bronchioloalveolar carcinoma: focal area of ground-glass attenuation at thin-section CT as an early sign. Radiology 199 (1996) 485-488

4. Kuriyama, K., Seto, M., Kasugai, T., Higashiyama, M., Kido, S., Sawai, Y., Kodama, K., Kuroda, D.: Ground-glass opacity on thin-section CT: value in differentiating subtypes of adenocarcinoma of the lung. Am. J. Roentgenol. 173 (1999) 465-469

5. Watanabe, S., Watanabe, T., Arai, K., Kasai, T., Haratake, J., Urayama, H.: Results of wedge resection for focal bronchioloalveolar carcinoma showing pure ground-glass attenuation on computed tomography. Ann. Thorac. Surg. 73 (2002) 1071-1075

6. Heitmann, K., Kauczor, H.U., Mildenberger, P., Uthmann, T., Perl, J., Thelen, M.: Automatic detection of ground glass opacities on lung HRCT using multiple neural networks. European Radiology 7 (1997) 1463-1472

7. Tanino, M., Takizawa, H., Yamamoto, S., Matsumoto, T., Tateno, Y., Iinuma, T.: A detection method of ground glass opacities in chest x-ray CT images using automatic clustering techniques. In: SPIE. Volume 5032. (2003) 1728-1737

8. Zhang, L., Fang, M., Naidich, D., Novak, C.: Consistent interactive segmentation of pulmonary ground glass nodules identified in CT studies. In: SPIE. Volume 5370. (2004) 1709-1719

9. Chang, S., Emoto, H., Metaxas, D., Axel, L.: Pulmonary micronodule detection from 3d chest CT. In: MICCAI. (2004) 821-828

10. Freund, Y., Schapire, R.: Experiments with a new boosting algorithm. In: the $13^{\text {th }}$ International Conference on Machine Learning. (1996) 148-156

11. Huang, X., Qian, Z., Huang, R., Metaxas, D.: Deformable-model based textured object segmentation. In: EMMCVPR. (2005) 119-135

12. Lorenz, C., Carlsen, I.C., Buzug, T., Fassnacht, C., Weese, J.: Multi-scale line segmentation with automatic estimation of width, contrast and tangential direction in 2d and 3d medical images. In: CVRMed-MRCAS. (1997) 233-42

13. Ali, S., Silvey, S.: A general class of coefficients of divergence of one distribution from another. J. Roy. Stat. Soc. 28 (1966) 131-142

14. Chernoff, H.: Large-sample theory: Parametric case. Ann. Math. Stat. 27 (1956) $1-22$

15. Zhou, J., Chang, S., Metaxas, D., Axel, L.: Vessel boundary extraction using ridge scan-conversion and the deformable model. In: ISBI. (2006) 189-192

16. Efron, B.: Estimating the error rate of a prediction rule: Improvement on crossvalidation. Journal of the American Statistical Association 78 (1983) 316-331 